\title{
Fast Enumeration of Combinatorial Objects
}

\author{
Boris Ryabko
}

\begin{abstract}
Summary . The problem of ranking (or perfect hashing) is well known in Combinatorial Analysis, Computer Science, and Information Theory. There are widely used methods for ranking permutations of numbers $\{1,2, \ldots, n\}, n \geq$ 1 , for ranking binary words of length $n$ with a fixed number of ones and for many other combinatorial problems. Many of these methods have nonexponential memory size and the time of enumeration $c_{1} n^{c_{2}}$ bit operations per letter, where $c_{1}>0, c_{2} \geq 1, n \rightarrow \infty$. In this paper we suggest a method which also uses non-exponential memory size and has the time of enumeration $O\left((\log n)^{\text {const }}\right)$ bit operations per letter, const $>0, n \rightarrow \infty$.
\end{abstract}

Index terms: fast ranking, enumerative encoding, perfect hashing.

\section{Introduction}

The problem of ranking can be described as follows. We have a set of combinatorial objects $S$, such as, say, the k-subsets of n things, and we can imagine that they have been arranged in some list, say lexicographically, and we want to have a fast method for obtaining the rank of a given object in the list. This problem is widely known in Combinatorial Analysis, Computer Science and Information Theory (see $[1,2]$ ). Ranking is closely connected with the hashing problem, especially with perfect hashing and with generating of random combinatorial objects. In Information Theory the ranking problem is closely connected with so-called enumerative encoding [3], which may be described as follows: there is a set of words $S$ and an enumerative code has to one-to-one encode every $s \in S$ by a binary word $\operatorname{code}(s)$. The length of the 
$\operatorname{code}(s)$ must be the same for all $s \in S$. Clearly, $|\operatorname{code}(s)| \geq \log |S|$. (Here and below $\left.\log x=\log _{2} x\right)$.)

The simplest method of coding is to store all words from $\mathrm{S}$ and all words code $(s), s \in S$, in the memory of the encoder and the decoder. In this case the time for encoding and decoding is proportional to $\log |S|$ and, obviously has a minimal value within a multiplicative constant when $|S|$ grows. However, the memory size of the encoder and decoder increases exponentially (as a function of the word length) just because they need to store all words $s \in S$ and $\operatorname{code}(s), s \in S$. Fortunately, for many interesting and important problems of enumeration there exist methods which do not use exponential memory size, see, for example, $[1,2]$. We consider two examples of such problems: enumeration of permutations and enumeration of the set of binary words with a given number of ones. These examples are well known in Combinatorial Analysis. Note that the second problem is very important for Information Theory where it forms the basis for many data compression codes. The first code, which does not use exponential memory size, was developed by Lynch [4], Davisson [5] and Babkin [6] (see also [2]). For this code the time of encoding and decoding per letter is more than const $\cdot n$ bit operations. This also holds for the time of encoding end decoding for known methods for ranking of permutations.

In this paper we suggest a new method for ranking (or enumerative encoding) for which the time of encoding and decoding is $O\left(\log ^{\text {const }} n\right)$ bit operations per letter. This method is based on the divide-and-conquer principle and uses the Schönhage-Strassen method of fast multiplication. As mentioned above, the proposed method is better than the known ones when there exists an algorithm with non-exponential memory size. The suggested method allows the exponential growth of the speed of encoding and decoding for all combinatorial problems of enumeration which are considered, for example, in [1] and [2] including the enumeration of permutations, compositions and others.

The next part describes the main idea of the proposed method. The descriptions of encoding and decoding are given in the parts 3 and 4, respectively. 


\section{The Main Idea}

The simplest but important example of the problem of ranking (and enumerative encoding) is the problem of integer translation from one radix to another. We will use this example to represent the main idea of the proposed method.

Consider the task of translation of an integer from a radix $m(m \geq 2)$ to the binary system. Let there be given an integer $x_{1} x_{2} \ldots x_{n}, n \geq 1$, in the number system $m$. A "common" method of translation is based on the following equality:

$$
\operatorname{code}\left(x_{1} \ldots x_{n}\right)=\sum_{i=1}^{n} x_{i} m^{n-i}
$$

Instead of this formula we can use the well- known Horner's scheme :

$$
\left.\operatorname{code}\left(x_{1} \ldots x_{n}\right)=\left(\ldots\left(x_{1} m+x_{2}\right) m+x_{3}\right) m+\ldots\right) m+x_{n}
$$

All calculations are performed in the binary system and as a result the $\operatorname{code}\left(x_{1} \ldots x_{n}\right)$ is the binary notation of the number $x_{1} x_{2} \ldots x_{n}$. Let us estimate the time required for calculation as in $(1)$. Here and below the time will be measured by the number of operations with single-bit words.

When calculating $\left(x_{1} m+x_{2}\right)$ we obtain a number of length $2\lceil\log m\rceil$ bits, and when calculating $\left(\left(x_{1} m+x_{2}\right) m+x_{3}\right)$, a number $3\lceil\log m\rceil$ bits long and so on. When we calculate these values we have at least to look through the words of length of $2\lceil\log m\rceil, 3\lceil\log m\rceil, \ldots, n\lceil\log m\rceil$. So it takes not less than $c n^{2} \log m$ bit operations to calculate $\operatorname{code}\left(x_{1} \ldots x_{n}\right)$ by $(1)$. So one can see that time per letter is not less than $c n \log m$.

The main idea of our approach is very simple. First we propose a new arrangement of brackets:

$$
\begin{aligned}
& \operatorname{code}\left(x_{1} \ldots x_{n}\right)=\left(\ldots\left(\left(x_{1} m+x_{2}\right)(m \cdot m)+\left(x_{3} m+x_{4}\right)\right)((m \cdot m)(m \cdot m))+\right. \\
& \left.+\left(\left(x_{5} m+x_{6}\right)(m \cdot m)+\left(x_{7} m+x_{8}\right)\right)+\ldots\right)
\end{aligned}
$$

When we use (2) most of the multiplications are carried out with short numbers. So the total time of calculation is small.

Secondly, we propose to use a fast method of multiplication in (2). We will use the Schönhage-Strassen method of multiplication which is the fastest one (see $[7,8]$ ). In this method the time $T(L)$ of multiplication of two binary 
numbers with $L$ digits (and the time of division of a number with $2 L$ digits by a number with $L$ digits) is given by

$$
T(L)=O(L \log L \log \log L), L \rightarrow \infty
$$

Let us estimate the time of calculations when (2) is used. Calculation of $(m \cdot m),\left(x_{1} m+x_{2}\right),\left(x_{3} m+x_{4}\right), \ldots,\left(x_{n-1} m+x_{n}\right)$ takes $(n / 2)+1$ multiplications of numbers with $\lceil\log m\rceil$ digits, calculation of $((m \cdot m)(m \cdot m)),\left(x_{1} m+x_{2}\right)(m$. $m)+\left(x_{3} m+x_{4}\right), \ldots,\left(x_{n-3} m+x_{n-2}\right)(m \cdot m)+\left(x_{n-1} m+x_{n}\right)$ takes $(n / 4)+1$ multiplications of numbers with $2\lceil\log m\rceil$ digits, and so on. Using this and the estimate $(3)$ we can see that the time of calculation of $\operatorname{code}\left(x_{1} \ldots x_{n}\right)$ by (2) is equal to

$$
\begin{aligned}
& O((n / 2)(\log m \log \log m \log \log \log m)+ \\
& (n / 4)(2 \log (2 m) \log \log (2 m) \log \log \log (2 m)+\ldots))= \\
& O\left(n \log ^{2} n \log \log n\right)
\end{aligned}
$$

So we can see that the time per letter is equal to $O\left(\log ^{2} n \log \log n\right)$.

Thus the proposed method takes $O\left(\log ^{2} n \log \log n\right)$ bit operations per letter instead of at least $n$ bit operations.

Note that our scheme is also valid for the task of calculation of values of any given polynomial.

Claim 1.Let $P(a)=y_{1} a^{n-1}+y_{2} a^{n-2}+\ldots+y_{n}$ be a polynomial and $y_{1}, y_{2}, \ldots y_{n}$ be integers, $m=\log \left(\max \left\{|a|,\left|y_{1}\right|, \ldots,\left|y_{n}\right|\right\}\right)$. The method of calculation of the value $P(a)$ according to the formula

$$
\begin{aligned}
& P(a)=\left(\left(\ldots\left(\left(y_{1} a+y_{2}\right)(a \cdot a)+\left(y_{3} a+y_{4}\right)\right)((a \cdot a)(a \cdot a))+\right.\right. \\
& \left.\left(y_{5} a+y_{6}\right)(a \cdot a)+\left(y_{7} \cdot a+y_{8}\right)\right) \ldots
\end{aligned}
$$

which uses the Schönhage-Strassen method of multiplication takes not more than $c \cdot n \cdot m \log ^{2}(n \cdot m) \log \log (n \cdot m)$ bit operations when $\mathrm{c}$ is constant, $n \rightarrow \infty$.

On the other hand, calculation by Horner scheme takes not less than const $\cdot\left(n^{2} \cdot m\right)$ bit operations.

The proposed simple idea will be used in this paper for fast ranking and enumerative coding for the general case. It is interesting that the method of "proper" arrangement of brackets is a special case of divide-and-conquer principle (see the definition in [7]). 


\section{$3 \quad$ Fast Ranking (or Encoding)}

Let $m \geq 2$ be an integer, $A=\left\{a_{1}, a_{2}, \ldots, a_{m}\right\}$ the alphabet and $A^{n}$ a set of words of length $n$ in the alphabet $A$, where $n \geq 0$ is an integer. Every $S \subset A^{n}$ is called a source. An enumerative code $\varphi$ is given by two mappings $\varphi^{c}: S \rightarrow$ $\{0,1\}^{t}$, where $t=\lceil\log |S|\rceil$ and $\varphi^{d}: \varphi^{c}(S) \rightarrow S$, so that $\varphi^{d}\left(\varphi^{c}(s)\right)=s$ for all $s \in S$ (here and below, $|x|$ is the cardinality of $x$ if $x$ is a set, and the length of $x$ if $x$ is a word). The map $\varphi^{c}$ is the encoder and the map $\varphi^{d}$ is the decoder. For the sake of simplicity we identify every word with a certain number from the interval $[0,1]$. For example, $0110=3 / 8$. Without loss of generality it is assumed that the alphabet $A$ is a set of integers from the interval $[0, m-1]$, and we may apply the lexicographic order to $A^{n}$.

Let us describe an enumerative code from [3]. Denote by $N_{s}\left(x_{1} \ldots x_{k}\right)$ the number of words which belong to $S$ and have the prefix $x_{1} \ldots x_{k}, k=$ $1,2, \ldots, n-1$. For $x_{1} x_{2} \ldots x_{n} \in S$ define

$$
\operatorname{code}\left(x_{1} \ldots x_{n}\right)=\sum_{i=1}^{n} \sum_{a<x_{i}} N_{S}\left(x_{1} \ldots x_{i-1} a\right)
$$

It is the code word for $x_{1} \ldots x_{n}$. It should be noted that there is a lot of interesting cases where the formula (4) allows to calculate the code using non-exponential memory size.

We give two examples of coding according to the formula (4). Both are taken from [1-3].

The first example is the enumeration of binary words with a given number

of ones. There is a source $S$ generating $n$-length binary words, $n>0$. There are $r, 0 \leq r \leq n$ ones in each word $x$.

It's easy to see that

$$
N_{s}\left(x_{1} \ldots x_{k-1} 0\right)=\left(\begin{array}{c}
n-k \\
r-\sum_{i=1}^{k-1} x_{i}
\end{array}\right)
$$

Using this formula and (4) we obtain

$$
\operatorname{code}\left(x_{1} \ldots x_{n}\right)=\sum_{k=1}^{n} x_{k}\left(\begin{array}{c}
n-k \\
r-\sum_{i=1}^{k-1} x_{i}
\end{array}\right)
$$

A time estimation of $c n \log n \log \log n(c>0)$ bit operations per letter is obtained in [2] for the problem of enumeration of binary words with a given number of ones. 
In the second example the enumeration of permutations is used. Let $A$ be $\{1,2, \ldots, n\}$. Given $x_{1} x_{2} \ldots x_{n}$ and $i, 1 \leq i \leq n, r_{i}$ denotes the number of integers which, first, are less than $x_{i}$, and, second, are situated to the right of $i$. The relation (4) becomes

$$
\operatorname{code}\left(x_{1} \ldots x_{n}\right)=\sum_{i=1}^{n} r_{i}(n-i) !
$$

Using Horner's scheme we obtain

$$
\left.\operatorname{code}\left(x_{1} \ldots x_{n}\right)=\left(\ldots\left(r_{1}(n-1)+r_{2}\right)(n-2)+r_{3}\right) \ldots\right)
$$

It is easy to estimate the time of calculation by (8) which is not less than $c n^{2}$ bit operations, where $c>0$ is constant. So the time per letter equals c $n$.

In order to describe the proposed method we consider a source $S \subset$ $A^{n}, n \geq 1$ and a word $x_{1} \ldots x_{n} \in S$.

Let us define

$$
\left.\begin{array}{l}
P\left(x_{1}\right)=N\left(x_{1}\right) /|S|, P\left(x_{k} / x_{1} \ldots x_{k-1}\right)=N\left(x_{1} \ldots x_{k}\right) / N\left(x_{1} \ldots x_{k-1}\right) \\
q\left(x_{1}\right)=\sum_{a<x_{1}} P(a), q\left(x_{k} / x_{1} \ldots x_{k}\right)=\sum_{a<x_{k}} P\left(a / x_{1} \ldots x_{k-1}\right), k=2, \ldots, n
\end{array}\right\}
$$

Clearly,

$$
\left.\begin{array}{l}
\sum_{i=1}^{n} \sum_{a<x_{i}} N\left(x_{1} \ldots x_{i-a} a\right)=|S|\left(q\left(x_{1}\right)+q\left(x_{2} / x_{1}\right) P\left(x_{1}\right)\right. \\
+q\left(x_{3} / x_{1} x_{2}\right) P\left(x_{2} / x_{1}\right) P\left(x_{1}\right)+q\left(x_{4} / x_{1} x_{2} x_{3}\right) P\left(x_{3} / x_{1} x_{2} P\left(x_{2} / x_{1}\right) P\left(x_{1}\right)+\ldots\right)
\end{array}\right\}
$$

From this equality and (4) we obtain

$$
\operatorname{code}\left(x_{1} \ldots x_{n}\right)=|S|\left(q\left(x_{1}\right)+q\left(x_{2} / x_{1}\right) P\left(x_{1}\right)+q\left(x_{3} / x_{1} x_{2}\right) P\left(x_{2} / x_{1}\right) P\left(x_{1}\right)+\ldots\right)
$$

In short, the proposed method may be described as follows: first, use the proper arrangement of brackets in (10) and, second, carry out all calculations using rational numbers. For the sake of simplicity we assume that $\log n$ is an integer. (In general case we can add, for example, the letters 0 to every word from $S$ in order to make $\log n$ an integer. It does not affect $|S|$ and the complexity of the code.) The formal implementation of the proper arrangement of brackets is:

$$
\left.\begin{array}{l}
\rho_{1}^{0}=P\left(x_{1}\right), \rho_{2}^{0}=P\left(x_{2} / x_{1}\right), \ldots, \rho_{n}^{0}=P\left(x_{n} / x_{1} x_{2} \ldots x_{n-1}\right) \\
\lambda_{1}^{0}=q\left(x_{1}\right), \lambda_{2}^{0}=q\left(x_{2} / x_{1}\right), \ldots, \lambda_{n}^{0}=q\left(x_{n} / x_{1} \ldots x_{n-1}\right)
\end{array}\right\}
$$




$$
\left.\begin{array}{l}
\rho_{k}^{s}=\rho_{2 k-1}^{S-1} \cdot \rho_{2 k}^{S-1}, s=1,2, \ldots, \log n ; k=1,2, \ldots, n / 2^{S} \\
\lambda_{k}^{S}=\lambda_{2 k-1}^{S-1}+\rho_{2 k}^{S-1} \cdot \lambda_{2 k}^{S-1}, s=1,2, \ldots, \log n ; k=1,2, \ldots, n / 2^{S}
\end{array}\right\}
$$

All calculations are carried out using rational numbers and all $\rho_{k}^{s}$ and $\lambda_{k}^{s}$ are fractions and presented as pairs of integers. The Shönhage-Strassen method is used for multiplications.

As a result we have

$$
\begin{aligned}
& \lambda_{1}^{\log n}=\left(q\left(x_{1}\right)+q\left(x_{2} / x_{1}\right) P\left(x_{1}\right)\right)+\left(q\left(x_{3} / x_{1} x_{2}\right)+\right. \\
& \left.q\left(x_{4} / x_{1} \ldots x_{3}\right) P\left(x_{3} / x_{1} x_{2}\right)\right) \cdot\left(P\left(x_{1}\right) P\left(x_{2} / x_{1}\right)\right)+\ldots
\end{aligned}
$$

We define the proposed code $\alpha^{c}$ as follows:

$$
\alpha^{c}\left(x_{1} \ldots x_{n}\right)=|S| \cdot \lambda_{1}^{\log n}
$$

Now let us consider some examples.

First, we consider the ranking of binary words with a given number of ones. Recall that

$$
\left(\begin{array}{c}
t \\
p
\end{array}\right)=\left(\begin{array}{c}
t-1 \\
p-1
\end{array}\right) \cdot \frac{t}{p}, \quad\left(\begin{array}{c}
t \\
p
\end{array}\right)=\left(\begin{array}{c}
t-1 \\
p
\end{array}\right) \cdot \frac{t}{t-p}
$$

Let $\Delta$ be 0 or 1 . Combining the last equalities, we obtain

$$
\left(\begin{array}{c}
t-1 \\
p-\Delta
\end{array}\right) /\left(\begin{array}{c}
t \\
p
\end{array}\right)=\frac{\Delta \cdot p+(1-\Delta)(t-p)}{t}
$$

This equality and (9), (5) yield

$$
\begin{gathered}
P\left(x_{t} / x_{1} \ldots x_{t-1}\right)=\frac{x_{t}\left(k-\sum_{j=1}^{t-1} x_{j}\right)+\left(1-x_{t}\right)\left(n-t+1-\left(k-\sum_{j=1}^{t-1} x_{j}\right)\right)}{n-t+1} \\
q\left(x_{t} / x_{1} \ldots x_{t-1}\right)=\frac{x_{t}\left(n-t+1-\left(k-\sum_{j=1}^{t-i} x_{j}\right)\right)}{n-t+1}
\end{gathered}
$$

$t=1,2, \ldots, n$.

Let us give an example. Let $n=8, k=3$ and the word $x_{1} x_{2} \ldots x_{8}=$ 01000101. From (14), (15) and (11), (12) we obtain

$$
p\left(x_{1}\right)=p(0)=\frac{0(3-0)+(1-0)(8-1+1-(3-0))}{8-1+1}=5 / 8
$$




$$
\begin{aligned}
& p\left(x_{2} / x_{1}\right)=p(1 / 0)=\frac{1(3-0)+(1-1)(8-1+1-(3-0))}{8-2+1}=3 / 7 \\
& p\left(x_{3} / x_{1} x_{2}\right)=p(0 / 01)=\frac{0(3-1)+(1-0)(8-3+1-(3-1))}{8-3+1}=4 / 6 \\
& p\left(x_{4} / x_{1} x_{2} x_{3}\right)=\frac{0(3-1)+(1-0)(8-4+1-(3-1))}{8-4+1}=3 / 5 \\
& p\left(x_{5} / x_{1} x_{2} x_{3} x_{4}\right)=p(0 / 0100)=\frac{0(3-1)+(1-0)(8-5+1-(3-1))}{8-5+1}=2 / 4 \\
& p\left(x_{6} / x_{1} \ldots x_{5}\right)=p(1 / 01000)=\frac{1(3-1)+0(8-6+1-(3-1))}{8-6+1}=2 / 3 \\
& p\left(x_{7} / x_{1} \ldots x_{6}\right)=p(0 / 010001)=\frac{0(3-2)+(1-0)(8-7+1-(3-2))}{8-7+1}=1 / 2 \\
& p\left(x_{8} / x_{1} \ldots x_{7}\right)=p(1 / 0100010)=\frac{1(3-2)+(1-1)(8-8+1-(3-2))}{8-8+1}=1 / 1 \\
& q\left(x_{1}\right)=q(0)=0 ; q=\left(x_{2} / x_{1}\right)=q(1 / 0)=\frac{1(8-2+1-(3-0))}{8-2+1}=4 / 7 \\
& q\left(x_{3} / x_{1} x_{2}\right)=q\left(x_{4} / x_{1} \ldots x_{3}\right)=q\left(x_{5} \ldots\right)=0 \\
& q\left(x_{6} / \ldots\right)=q(1 / 01000)=\frac{1(8-6+1-(3-1))}{8-6+1}=1 / 3 \\
& q\left(x_{7} / \ldots\right)=0, q\left(x_{8} / \ldots\right)=q(1 / 0100010)=\frac{1(8-8+1-(3-2))}{8-8+1}=0 \\
& \rho_{1}^{0}=5 / 8, \rho_{2}^{0}=3 / 7, \rho_{3}^{0}=4 / 6, \rho_{4}^{0}=3 / 5, \rho_{5}^{0}=2 / 4, \rho_{6}^{0}=2 / 3, \rho_{7}^{0}=1 / 2, \rho_{8}^{0}=1 \\
& \lambda_{1}^{0}=0, \lambda_{2}^{0}=4 / 7, \lambda_{3}^{0}=0, \lambda_{4}^{0}=0, \lambda_{5}^{0}=0, \lambda_{6}^{0}=1 / 6, \lambda_{7}^{0}=0, \lambda_{8}^{0}=0 \\
& \rho_{1}^{1}=5 / 8 \cdot 3 / 7, \rho_{2}^{1}=4 / 6 \cdot 3 / 5, \rho_{3}^{1}=2 / 4 \cdot 2 / 3, \rho_{4}^{1}=1 / 2 \cdot 1 / 2 \cdot 1 \\
& \lambda_{1}^{1}=0+5 / 8 \cdot 4 / 7, \lambda_{2}^{1}=0+0, \lambda_{3}^{1}=0+1 / 3 \cdot 2 / 4, \lambda_{3}^{1}=0+\cdot 1 / 3 \cdot 2 / 4, \lambda_{4}^{1}=0+0 \\
& \rho_{1}^{2}=5 / 8 \cdot 3 / 7 \cdot 4 / 3 \cdot 3 / 5 ; \rho_{2}^{2}=2 / 4 \cdot 2 / 3 \cdot 1 / 2 \cdot 1 / 1 \\
& \lambda_{1}^{2}=5 / 8 \cdot 4 / 7+5 / 8 \cdot 3 / 7 \cdot 0=5 / 8 \cdot 4 / 7 \\
& \lambda_{2}^{2}=1 / 3 \cdot 2 / 4+2 / 4 \cdot 2 / 3 \cdot 0=1 / 3 \cdot 2 / 3 \\
& \lambda_{1}^{3}=5 / 8 \cdot 4 / 7+5 / 8 \cdot 3 / 7 \cdot 4 / 6 \cdot 3 / 5 \cdot 1 / 3 \cdot 2 / 4=20 / 56+1 / 56=21 / 56
\end{aligned}
$$


Observe that there are $\left(\begin{array}{l}8 \\ 3\end{array}\right)=56$ binary words of the length 8 with 3 ones. Thus, from (13) we obtain a code word:

$$
\alpha^{c}(01000101)=56 \cdot(21 / 56)=21
$$

Of course, calculations according to the formula (6) give the same result: code $(01000101)=21$. (For the sake of clearness we carry out all calculations with decimal numbers instead of binary ones).

Let us consider the enumeration of permutations. From the definition we obtain $N_{s}\left(x_{1} \ldots x_{k}\right)=(n-k)$ !; see also (7). The equalities (9), (11)-(13) yield

$$
\begin{gathered}
\alpha^{c}\left(x_{1} x_{2} \ldots x_{n}\right)= \\
n !\left(\left(\frac{r_{1}}{n}+\frac{r_{2}}{n \cdot(n-1)}\right)+\left(\frac{1}{n} \cdot \frac{1}{n-1}\right)\left(\frac{r_{3}}{n-2}+\frac{r_{4}}{(n-2)(n-3}\right)+\right. \\
+\left(( ( \frac { 1 } { n } \cdot \frac { 1 } { n - 1 } ) \cdot ( \frac { 1 } { n - 2 } \cdot \frac { 1 } { n - 3 } ) ) \left(\left(\frac{r_{5}}{n-4}+\frac{r_{6}}{(n-2)(n-5)}\right)+\right.\right. \\
\left.+\left(\frac{1}{(n-4)} \cdot \frac{1}{(n-5)}\right)\left(\frac{r_{7}}{n-6}+\frac{r_{8}}{(n-6)(n-7)}\right)+\ldots\right)
\end{gathered}
$$

In order to estimate the complexity of the method $\alpha^{c}$ we define several values. Let, as before, $S \subset A^{n}$ be given. By definition $T$ is the maximal time (in bit operations) for calculation of rational fractions $N\left(x_{1} \ldots x_{t+1}\right) / N\left(x_{1} \ldots x_{t}\right)$, where $x_{1} \ldots x_{n} \in S, t=1,2, \ldots, n-1, M$ is the size (in bits) of the program that is used to compute

$$
\left\{N\left(x_{1} \ldots x_{t-1}\right) / N\left(x_{1} \ldots x_{t}\right) ; x_{1} \ldots x_{n} \in S, t=1,2, \ldots n-1\right\}
$$

and let $Q$ be the maximal denominator of rational fractions

$$
N\left(x_{1} \ldots x_{t+1}\right) / N\left(x_{1} \ldots x_{t}\right), x_{1} \ldots x_{n} \in S, t=1, \ldots, n .
$$

Let us define

$$
\hat{Q}=\max \{|A|, Q\}
$$

Theorem 1. Let there be given an alphabet $A$, an integer $n$ and $S \subset A^{n}$. The proposed method of encoding $\alpha^{c}$ has the following properties:

i) $\alpha^{c}$ is correct, i.e. for every $x, y \in S \alpha^{c}(x) \neq \alpha^{c}(y)$ and $\alpha^{c}(x)$ is an integer from $[0,|S|-1]$ 
ii) the time of encoding per letter is

$$
T+O(\log n \log \hat{Q} \log (n \log \hat{Q}) \log \log (n \log \hat{Q}))
$$

\section{bit operations}

iii) the memory size of the encoder is $M+O(n \log \hat{Q} \log n)$ bits.

Proof. The claim i) immediately follows from (10)-(13).

For the sake of simplicity of the proof of ii) we assume that $\log n$ and $\log \hat{Q}$ are integers. According to the definition of $\hat{Q}$ and (14), (15) we can see that the notation of every $P()$ and $q()$ uses $2 \log \hat{Q}$ bits $(\log \hat{Q}$ bits for the numerator and $\log \hat{Q}$ bits for the denominator). That is why the calculation of $\rho_{k}^{1}, k=1,2, \ldots, n / 2$ according to $(12)$ takes $2(n / 2)$ multiplications of numbers of the length $\log \hat{Q}$ bits and the calculation of $\lambda_{k}^{1}, k=1, \ldots, n / 2$ according to (12) and the formula $a / b+c / d=(a d+b c) /(b d)$ takes $3(n / 2)$ multiplications of numbers with the length $\log \hat{Q}$ bits. The calculations of $\rho_{k}^{2}, \lambda_{k}^{2}, k=1,2, \ldots, n / 4$ take $5(n / 4)$ multiplications of numbers of the length $2 \log \hat{Q}$ bits each. Similarly, the calculation of $\rho_{k}^{i}, \lambda_{k}^{i}, k=1,2, \ldots, n / 2^{i}$ takes $5\left(n / 2^{i}\right)$ multiplications of numbers with the length $2^{i} \log n$ bits. From (3) we obtain that the general time of calculations is:

$$
\begin{aligned}
& (5 n / 2) O(\log \hat{Q} \log \log \hat{Q} \log \log \log \hat{Q})+ \\
& (5 n / 4) O(2 \log \hat{Q} \log (2 \log \hat{Q}) \log \log (2 \log \hat{Q})+\ldots \\
& \left(5 n / 2^{i}\right) O\left(2^{i} \log \hat{Q} \log \left(2^{i} \log \hat{Q}\right) \log \log \left(2^{i} \log \hat{Q}\right)+\right. \\
& \ldots+5 \cdot O(n \log \hat{Q} \log (n \log \hat{Q}) \log \log (n \log \hat{Q})
\end{aligned}
$$

It is easy to see that the last value is not more than

$$
O(n \log n \log \hat{Q}) \log (n \log \hat{Q}) \log \log (n \log \hat{Q}))
$$

bit operations. It yields

$$
O(\log n \log \hat{Q} \log (n \log \hat{Q}) \log \log (n \log \hat{Q}))
$$

bit operations per letter for calculation of $\lambda_{1}^{\log n}$. In order to obtain $\lambda^{c}\left(x_{1} \ldots x_{n}\right)$ we should calculate the product $|S| \lambda_{1}^{\log n}$, see (13). $S$ is a subset of $A^{n}$, so $|S| \leq|A|^{n}$ and a binary notation of the numbers $|S|$ and $\lambda_{1}^{\log n}$ takes not more than $n \cdot \log |A|$ bits. From (3) we can see that the time of calculation 
of $|S| \lambda_{1}^{n}$ is equal to $O(n \log |A| \log (n|A|) \log \log (n|A|)$ bit operations per letter. From this, (17), and (16) we obtain ii).

In order to estimate the size of the encoder program, note that when it calculates $\lambda_{k}^{i}, \rho_{k}^{i}$ it can store only $\lambda_{k}^{i-1}, \rho_{k}^{i-1}, i=2, \ldots, \log n$, the same memory is used to store $\left\{\lambda_{k}^{i-1}, \rho_{k}^{i-1} ; k=1, \ldots, n / 2^{i-1}\right\}$ and $\left\{\lambda_{k}^{i}, \rho_{k}^{i} ; k=1, \ldots, n / 2^{i}\right\}$. From this and the definitions of $m$ and $\hat{Q}$ we can easily obtain iii). Theorem 1 is proved.

\section{Fast Decoding}

First, we describe the general scheme of decoding not taking into account the time of calculation. Let an alphabet $A=\{0,1, \ldots, m-1\}$ and a source $S \subset A^{n}$ be given and let $\hat{x}=x_{1} x_{2} \ldots x_{n}$ be a word from $S$ and $y=\alpha^{c}(\hat{x})$ be the encoded word $\hat{x}$.

In order to decode $\hat{y}$ we consider $y_{1}=y /|S|$ as a rational number and first find $i_{1}$ satisfying the inequalities

$$
\lambda_{i_{1}}^{0} \leq \hat{y}_{1}<\lambda_{i_{1}+1}^{0}
$$

From these inequalities it follows that the first letter of the encoded word is $i_{1}: x_{1}=i_{1}$. After that we calculate

$$
\hat{z}=\left(y_{1}-\lambda_{i_{1}}^{0}\right) / \rho_{i_{1}}^{0}
$$

where $\hat{z}$ is a rational number, and find $i_{2}$ complying with the inequalities

$$
\lambda_{i_{2}}^{0} \leq \hat{z}<\lambda_{i_{2}+1}^{0}
$$

If follows that the second letter is $i_{2}$.

Of course, we could use this way to find the third letter, then the fourth one, etc. But we use a more complicated way which will give a possibility to operate with short numbers. We calculate $\lambda_{1}^{1}$ according to (12). (It is possible because now $x_{1}$ and $x_{2}$ are known now.) After that we calculate

$$
y_{2}=\left(y_{1}-\lambda_{1}^{1}\right) / \rho_{1}^{1}
$$

and find letters $x_{3}, x_{4}$ using $y_{2}$ as we have found $x_{1}, x_{2}$ using $y_{1}$. Then we calculate $\lambda_{2}^{1}$ using $x_{3}$ and $x_{4}$ and $\lambda_{1}^{2}$ using $\lambda_{1}^{1}, \lambda_{2}^{1}$ and $\rho_{1}^{1}$, see (12). And so on. 
The point is that when we carry out calculations (18)-(20) we can use only estimations of $y_{1}, y_{2}, \lambda_{i}^{1}, \rho_{i}^{1}$, etc, which are based on the few leading digits. More exactly, we will use two estimations for every value which are an upper bound and a lower one.

In order to give the exact definition, first we define several auxiliary values. Let $p / q$ be a rational number represented as a pair of the integers $p, q$, $0<p \leq q$, and let $t \geq 1$ be an integer. We define two functions $\varphi_{t}^{+}(p / q)$ and $\varphi_{t}^{-}(p / q)$ as follows. Let $l=\lfloor\log q\rfloor$, and $\left(q_{l} q_{l-1} \ldots q_{0}\right)$ and $\left(p_{l} \ldots p_{0}\right)$ be binary representations of $q$ and $p$, correspondingly. Then

$$
\begin{aligned}
& \varphi_{t}^{+}(p / q)=\left(\sum_{i=l-t+1}^{l} p_{i} 2^{i}+2^{l-t}\right) /\left(\sum_{i=l-t+1}^{l} q_{i} 2^{i}\right) \\
& \varphi_{t}^{-}(p / q)=\left(\sum_{i=l-t+1}^{l} p_{i} 2^{i}\right) /\left(\sum_{i=l-t+1}^{l} q_{i} 2^{i}+2^{l-t}\right)
\end{aligned}
$$

For example, $\varphi_{3}^{+}(5 / 17)=3 / 8, \varphi_{3}^{-}(5 / 17)=2 / 9$.

We will need the following simple bounds.

Lemma. Let $p, q, t$ be integers, $0<p \leq q, t>2$. Then

$$
\begin{aligned}
& 0 \leq \varphi_{t}^{+}(p / q)-p / q<2^{2-t} \\
& 0 \leq p / q-\varphi_{t}^{-}(p / q)<2^{2-t}
\end{aligned}
$$

Proof. It's easy to see that if $x<1 / 2$ then

$$
\frac{1}{1-x}<1+2 x, \frac{1}{1+x}>1-x
$$

Theae bounds immediately follow from well known equalities

$$
\begin{gathered}
(1-x)^{-1}=1+x+x^{2}+\ldots=1+x+x^{2} /(1-x)=1+x(1+x /(1-x)) \\
(1+x)^{-1}=1-x+x^{2}-\ldots=1-x+x^{2}\left(1-x+x^{2}-\ldots\right)
\end{gathered}
$$

The following sequence of inequalities gives the bound (21):

$$
\begin{gathered}
\varphi_{t}^{+}(p / q) \leq \frac{p+2^{l-t}}{q-2^{l-t}}<p / q\left(1+2^{l-t} / p\right)\left(1+2 \cdot 2^{l-t} / q\right)= \\
p / q+2^{l-t} / q+2 \cdot 2^{l-t} / q+2^{l-t} / q \cdot 2 \cdot \cdot^{l-t} / q<p / q+2^{-t}+
\end{gathered}
$$




$$
2 \cdot 2^{-t}+2^{-2 t}<p / q+4 \cdot 2^{-t}
$$

Here we use (23) and the obvious inequality $2^{l} \leq q$.

Let us proceed with the description of the method of decoding. Let, as before, an alphabet $A=\{0,1, \ldots, m-1\}$ and a source $S \subset A^{n}$ be given.

As before, let $Q$ be the maximal denominator of the rational numbers $N\left(x_{1} \ldots x_{t+1}\right) / N\left(x_{1} \ldots x_{t}\right), x_{1} \ldots x_{t+1} \in S, t=1,2, \ldots, n-1$. From this definition and (11), (12) we immediately obtain that the denominators of the rational fractions $\rho_{i}^{s}$ and $\lambda_{i}^{s}$ not exceeding $Q^{s}$, for all $s=1, \ldots, \nu ; k=1, \ldots, n / 2^{s}$. Let

$$
h=\lceil\log Q\rceil+3
$$

We will give the definition by induction on $n$. First, let $n=2$. For every value $\lambda_{j}^{i}$ we define the upper and the lower estimations, $\lambda^{+}(i, j)$ and $\lambda^{-}(i, j)$.

Let the decoder calculate

$$
\begin{gathered}
\lambda^{+}(1,1)=\varphi_{2 h}^{+}(y /|S|), \quad \lambda^{-}(1,1)=\varphi_{2 h}^{-}(y /|S|) \\
\lambda^{+}(0,1)=\varphi_{h}^{+}\left(\lambda^{+}(1,1)\right), \quad \lambda^{-}(0,1)=\varphi_{h}^{-}\left(\left(\lambda^{-}(1,1)\right)\right.
\end{gathered}
$$

Then it finds $i_{1}$ complying with the inequalities

$$
q\left(i_{1}\right) \leq \lambda^{+}(0,1), q\left(i_{1}+1\right)>\lambda^{-}(0,1)
$$

We use these inequalities instead of (23). But here the decoder carries out calculations with $(h+3)$ - length words instead of the whole binary notations of $y$ and $|S|$. The inequalities (27) mean that the first letter of coded words is $i_{1}: x_{1}=i_{1}$. Let us define

$$
\begin{aligned}
& \lambda^{+}(0,2)=\varphi_{h}^{+}\left(\left(\lambda^{+}(1,1)-\lambda_{1}^{0}\right) / \rho_{1}^{0}\right) \\
& \lambda^{-}(0,2)=\varphi_{h}^{-}\left(\left(\lambda^{-}(1,1)-\lambda_{1}^{0}\right) / \rho_{1}^{0}\right)
\end{aligned}
$$

and find $i_{2}$ complying with inequalities

$$
q\left(i_{2}\right) \leq \lambda^{+}(0,2), \quad q\left(i_{2}+1\right)>\lambda^{-}(0,2)
$$

It means that the second letter is $i_{2}: x_{2}=i_{2}$.

Let now $n$ be greater than 2. In order to use the divide-and-conquer principle we define

$$
\begin{gathered}
\lambda^{+}(\lceil\log n\rceil, 1)=\varphi_{n h}^{+}(y /|S|) \\
\lambda^{-}(\lceil\log n\rceil, 1)=\varphi_{n h}^{-}(y /|S|) \\
\lambda^{+}(\lceil\log n\rceil-1,1)=\varphi_{\lceil n h / 2\rceil}^{+}\left(\lambda_{-}^{+}(\lceil\log n\rceil, 1)\right) \\
\lambda^{-}(\lceil\log n\rceil-1,1)=\varphi_{\lceil n h / 2\rceil}^{-}(\lambda(\lceil\log n\rceil, 1))
\end{gathered}
$$


Then the decoder finds $x_{1}, x_{2}, \ldots, x_{\lceil n / 2\rceil}$ using $\lambda^{+}(\lceil\log n\rceil-1,1), \lambda^{-}(\lceil\log n\rceil-$ $1,1)$ and calculates $\lambda_{1}^{\lceil\log n\rceil-1}$ (see $(11),(12)$ ). After that the decoder calculates

$$
\begin{aligned}
& \lambda^{+}(\lceil\log n\rceil-1,2)=\varphi_{\lceil n h / 2\rceil}^{+}\left(\left(\lambda^{+}(\lceil\log n\rceil, 1)-\lambda_{1}^{\lceil\log n\rceil-1}\right) / \rho_{1}^{\lceil\log n\rceil-1}\right) \\
& \lambda^{-}(\lceil\log n\rceil-1,2)=\varphi_{\lceil n h / 2\rceil}^{-}\left(\left(\lambda^{-}(\lceil\log n\rceil, 1)-\lambda_{1}^{\lceil\log n\rceil-1}\right) / \rho_{1}^{\lceil\log n\rceil-1}\right)
\end{aligned}
$$

and uses this pair in order to find $x_{\lceil n / 2\rceil+1}, x_{\lceil n / 2\rceil+2}, \ldots, x_{n}$. So (29) and (30) give a possibility to decode the $n$-letter word as two words of length $\lceil n / 2\rceil$ and $(n-\lceil n / 2\rceil)$, correspondingly.

In order to give an example of the decoding, let us consider the previous example. Let, as before, $S$ be a set of all binary words of length 8 and each of them has 3 ones. Let the proposed method be applied for decoding of the word $010101=(21)_{10}$.

According to the description, first, the decoder finds

$$
\begin{aligned}
& \lambda^{+}(\lceil\log n\rceil, 1)=\lambda^{+}(3,1)=\varphi_{48}^{+}(25 / 56)=\left(21 \cdot 2^{42}+1 / 56 \cdot 2^{42}\right) \\
& \lambda^{-}(\lceil\log n\rceil, 1)=\lambda^{-}(3,1)=\varphi_{48}^{-}(25 / 56)=\left(21 \cdot 2^{42} / 56 \cdot 2^{42}+1\right)
\end{aligned}
$$

and

$$
\begin{gathered}
\lambda^{+}(2,1)=\varphi_{21}^{+}\left(21 \cdot 2^{42}+1 / 56 \cdot 2^{42}\right)=\left(21 \cdot 2^{23}+1 / 56 \cdot 2^{23}\right) \\
\lambda^{-}(2,1)=\left(21 \cdot 2^{23} / 56 \cdot 2^{23}+1\right)
\end{gathered}
$$

Using $\lambda^{+}(2,1)$ and $\lambda^{-}(2,1)$ the decoder should find $x_{1}, x_{2}, x_{3}, x_{4}$. According to the algorithm, it calculates

$$
\begin{aligned}
& \lambda^{+}(1,1)=\varphi_{12}^{+}\left(21 \cdot 2^{23}+1 / 56 \cdot 2^{23}\right)=\left(21 \cdot 2^{7}+1 / 56 \cdot 2^{7}\right) \\
& \lambda^{-}(1,1)=\left(21 \cdot 2^{7} / 56 \cdot 2^{7}+1\right)
\end{aligned}
$$

This pair encodes $x_{1}, x_{2}$. After that the decoder finds

$$
\begin{aligned}
& \lambda^{+}(0,1)=\varphi_{6}^{+}\left(21 \cdot 2^{7}+1 / 56 \cdot 2^{7}\right)=22 / 56 \\
& \lambda^{-}(0,1)=\varphi_{6}^{-}\left(21 \cdot 2^{7}+1 / 56 \cdot 2^{7}\right)=21 / 57
\end{aligned}
$$

which encode $x_{1}$. For given $S q(0)=0, q(1)=5 / 8$ (see the example of coding), $x_{1}=0$ because

$$
0=q\left(i_{1}\right) \leq \lambda^{+}(0,1)=22 / 56
$$




$$
21 / 57=\lambda^{-}(0,1)<q\left(i_{1}+1\right)=1
$$

(see $(22))$. According to $(24)$ the decoder calculates

$$
\begin{gathered}
\lambda^{+}(0,2)=\varphi_{6}^{+}\left(\left(21 \cdot 2^{6}+1 / 56 \cdot 2^{7}-0\right) /(5 / 8)=169 / 280\right. \\
\lambda^{-}(0,2)=168 / 281
\end{gathered}
$$

Thus, $x_{2}=1$ because $q(0 / 0)=0, q(1 / 0)=4 / 7$ and, obviously,

$$
4 / 7 \leq 169 / 280=\lambda^{+}(0,2), \lambda^{-}(0,2)=168 / 281<1 .
$$

After finding $x_{1}=0$ and $x_{2}=1$ the decoder calculates $\lambda_{1}^{1}=5 / 14, \rho_{1}^{1}=15 / 56$ and finds $\lambda^{+}(1,2), \lambda^{-}(1,2)$ according to $(24)$. It gives $x_{3}=0, x_{4}=0$ and so on.

The next theorem characterizes properties of the proposed method of decoding which we denote as $\alpha^{d}$.

Theorem 2. Let there be an alphabet $A$, an integer $n$ and $S \subset A^{n}$. Then the proposed method of decoding $\alpha^{d}$ has the following properties:

i) $\alpha^{d}$ is correct, i.e. for every $x \in S$

$$
\alpha^{d}\left(\alpha^{c}(x)\right)=x
$$

ii) the time of decoding per letter is

$$
T+O(\log \hat{Q}(\log n \log (n \hat{Q})) \log \log (n \hat{Q})
$$

iii) the memory size of the decoder is

$$
M+O(n \log \hat{Q} \log n)
$$

(here T, $M$ and $\hat{Q}$ are defined as in Theorem 1.)

Proof. First, we estimate the speed of decoding. As it follows from the algorithm every operation of multiplication for calculation of $\lambda_{j}^{i}$ corresponds to two divisions when the decoder calculates $\lambda^{+}(i, j)$ and $\lambda^{-}(i, j)$ according to (28)-(30). The time of divisions in (28)-(30) is proportional to the time of multiplications, see (12). So the time of calculations of $\lambda^{+}(i, j)$ and $\lambda^{-}(i, j)$ is equal to the time of calculations of $\lambda_{j}^{i}$ within a multiplicative constant. It is easy to see that the time of finding $i_{1}=x_{1}, i_{2}=x_{2} \ldots$, according to (27) does not change the asymptotical estimation of the time of decoding. 
Let us estimate the memory size. For this purpose we note that the decoder can use the same memory size for decoding the first letters $x_{1} \ldots x_{\lceil n / 2\rceil}$ and the letters $x_{\lceil n / 2\rceil+1}, \ldots, x_{n}$. From this fact it immediately follows that the decoder can use such a memory size as does the encoder and it gives the same estimation for the memory size.

Now we will show that $\alpha^{d}$ is a correct method of decoding. For this purpose for a letter $x_{j}, j=1, \ldots n$, we estimate the values

$$
\left|\lambda^{+}(0, j)-q\left(x_{j}\right)\right| \text { and }\left|q\left(x_{j}\right)-\lambda^{-}(0, j)\right|
$$

It is important because the decoder decides that letter $x_{j}$ sould be decoded as $i_{j}$ if the inequalities

$$
q\left(i_{j}\right) \leq \lambda^{+}(0, j), \quad q\left(i_{j}+1\right) \geq \lambda^{-}(0, j)
$$

are valid. As it follows from (11), the (31) are equal to

$$
\lambda_{i_{j}}^{0} \leq \lambda^{+}(0, j), \quad \lambda_{i_{j}+1}^{0} \geq \lambda^{-}(0, j)
$$

and these inequalities should be valid for one $i_{j}$ in the case of $x_{j}=i_{j}$. And this property should be valid for all letters $x_{1}, x_{2}, \ldots, x_{n}$ and for all $x_{1} \ldots x_{n} \in S$.

By definition $Q$ is the maximal denominator of the rational fractions

$$
P\left(x_{t+1} / x_{1} \ldots x_{t}\right)=N\left(x_{1} \ldots x_{t+1}\right) / N\left(x_{1} \ldots x_{t}\right)
$$

$t=1, \ldots, n-1 ; x_{1} \ldots x_{n} \in S$. It means that for every $x_{1} \ldots x_{t}, i, j \in A ; i \neq j$.

$$
\begin{gathered}
\left|q\left(i / x_{1} \ldots x_{t}\right)-q\left(j / x_{1} \ldots x_{t}\right)\right| \geq 1 / Q \\
q\left(i / x_{1} \ldots x_{t}\right) \geq 1 / Q
\end{gathered}
$$

see (9). From the definition (11) we obtain

$$
\left|\lambda_{i}^{0}-\lambda_{j}^{0}\right| \geq 1 / Q
$$

From this inequality and (32) we can see that the inequalities

$$
\begin{aligned}
& 0 \leq \lambda^{+}(0, j)-\lambda_{j}^{0}<1 / Q \\
& 0 \leq \lambda_{j}^{0}-\lambda^{-}(0, j)<1 / Q
\end{aligned}
$$

guarantee the correctness of decoding. We will prove only the first pair of inequalities because the second one can be proved in the same way. We will 
investigate the value $\lambda^{+}(0, n)-\lambda_{n}^{0}$ because it can be easily seen from a proof that the possible error is maximal for the last letter $x_{n}$. First, we notice that the inequality

$$
\lambda^{+}(0, j)-\lambda_{j}^{0} \geq 0
$$

is immediately obtained from the definition of $\varphi^{+}()$and $\lambda^{+}($), see (25)-(30). Now we have to prove that

$$
\lambda^{+}(0, n)-\lambda_{n}^{0}<1 / Q
$$

We define

$$
\left.\varepsilon(i, j)=\lambda^{+}(i, j)-\left(\lambda^{+}(i+1, j / 2)-\lambda_{j-1}^{i}\right) / \rho_{j-1}^{i}\right)
$$

$i=0, \ldots, j=n / 2^{i}$. In fact, $\varepsilon(i, j)$ is an error arising from using $\varphi_{2^{i h}}^{+}\left(\left(\lambda^{+}(i+\right.\right.$ $\left.\left.1, j / 2)-\lambda_{j-1}^{i}\right) \rho_{j-1}^{i}\right)$ instead of the value $\left(\lambda^{+}(i+1, j / 2)-\lambda_{j-1}^{i}\right) / \rho_{j-1}^{i}$. The following train of expressions is valid:

$$
\begin{aligned}
& \lambda^{+}(0, n)=\varphi_{h}^{+}\left(\left(\lambda^{+}(1, n / 2)-\lambda_{n-1}^{0}\right) / \rho_{n-1}^{0}=\varepsilon(0, n)+\left(\lambda^{+}(1, n / 2)-\lambda_{n-1}^{0}\right) / \rho_{n-1}^{0}=\right. \\
& \varepsilon(0, n)+\left(\varphi_{2 h}^{+}\left(\left(\lambda^{+}(2, n / 4)-\lambda_{n / 2-1}^{1}\right) / \rho_{n / 2-1}^{1}\right)-\lambda_{n-1}^{0}\right) / \rho_{n-1}^{0}= \\
& \varepsilon(0, n)+\left(\left(\varepsilon(1, n / 2)+\left(\lambda^{+}(2, n / 4)-\lambda_{n / 2}^{1}\right) / \rho_{n / 2-1}^{1}\right)-\lambda_{n-1}^{0}\right) / \rho_{n-1}^{0}= \\
& =\varepsilon(0, n)+\varepsilon(1, n / 2) / \rho_{n-1}^{0}+\left(\left(\varphi_{4 h}^{+}\left(\lambda^{+}(3, n / 8)-\lambda_{n / 4-1}^{2}\right) / \rho_{n / 4-1}^{2}-\lambda_{n / 2}^{1}\right) /\right. \\
& \left.\left.\rho_{n / 2-1}^{1}\right)-\lambda_{n-1}^{0}\right) \rho_{n-1}^{0}=\varepsilon(0, n)+\varepsilon(1, n / 2) / \rho_{n-1}^{0}+\varepsilon(2, n / 4) /\left(\rho_{n-1}^{0} \cdot \rho_{n / 2}^{1}\right)+ \\
& \left.\left.\left(\ldots\left(\varphi_{8 h}^{+}\left(\lambda^{+}\left(4, n / 2^{4}\right)-\lambda_{n / 8-1}^{3}\right) / \rho_{n / 8-1}^{3}-\lambda_{n / 4-1}^{2}\right) / \rho_{n / 4-1}^{2}-\lambda_{n / 2}^{1}\right) / \rho_{n / 2-1}^{1}\right)-\lambda_{n-1}^{0}\right) / \rho_{n-1}^{0} \\
& =\ldots=\varepsilon(0, n)+\varepsilon(1, n / 2) / \rho_{n-1}^{0}+\varepsilon(2, n / 4) /\left(\rho_{n-1}^{0} \cdot \rho_{n / 2-1}^{1}\right)+\varepsilon(3, n / 8) / \\
& \left(\rho_{n-1}^{0} \cdot \rho_{n / 2-1}^{1} \cdot \rho_{n / 4-1}^{2}\right)+\ldots+\varepsilon(\log n, 1) /\left(\rho_{n-1}^{0} \cdot \rho_{n / 2-1}^{1} \ldots \rho_{1}^{\log n-1}\right)+ \\
& \left.+\left(\left(\ldots\left(\lambda_{1}^{\log n}-\lambda_{1}^{\log n-1}\right) / \rho_{1}^{\log n-1}-\lambda_{3}^{\log -2}\right) / \rho_{3}^{\log n-2}-\lambda_{7}^{\log n-3}\right) / \rho_{7}^{\log n-3}-\ldots\right) / \rho_{n-1}^{0}
\end{aligned}
$$

So we obtain

$$
\begin{gathered}
\lambda^{+}(0, n)=\sum_{i=0}^{\log n} \varepsilon\left(i, n \cdot 2^{-i}\right) / \prod_{j=0}^{i=1} \rho_{n 2^{-j}-1}^{j}+ \\
\left.+\left(\ldots\left(\lambda_{1}^{\log n}-\lambda_{1}^{\log n-1}\right) / \rho_{1}^{\log n-1}-\lambda_{3}^{\log n-2}\right) / \rho_{3}^{\log n-2}-\ldots\right) / \rho_{n-1}^{0}
\end{gathered}
$$


Using the definition (12) of $\lambda_{j}^{i}$ we obtain

$$
\lambda^{+}(0, n)=\sum_{i=0}^{\log n} \varepsilon\left(i, n 2^{-i}\right) / \prod_{i=0}^{i-1} \rho_{n 2^{-j}-1}^{j}+\lambda_{n}^{0}
$$

Thus,

$$
\lambda^{+}(0, n)-\lambda_{n}^{0}=\sum_{i=0}^{\log n} \varepsilon\left(i, n 2^{-i}\right) / \prod_{i=0}^{i-1} \rho_{n 2^{-j}-1}^{j}
$$

This equality and (33), (12) yield

$$
\begin{gathered}
\lambda^{+}(0, n)-\lambda_{n}^{0} \leq \varepsilon(0, n)+\varepsilon(1, n / 2) Q+\varepsilon(2, n / 4) Q^{3}+\varepsilon(3, n / 8) Q^{7}+ \\
\ldots+\varepsilon(\log n, 1) Q^{2^{n}-1}
\end{gathered}
$$

The claim of the Lemma, (28), (29) and (35) yield

$$
\varepsilon(i, j)<2^{2-2^{i} h}
$$

The last two inequalities and (24) give us

$$
\lambda^{+}(0, n)-\lambda_{n}^{0}<4\left(1 / 8 Q+Q /(8 Q)^{2}+Q^{3} /(8 Q)^{4}+\ldots\right)
$$

Hence, we obtain the inequality

$$
\lambda^{+}(0, n)-\lambda_{n}^{0}<1 / Q
$$

which completes the proof of (34). Theorem 2 is proved. 


\section{References}

1. Reingold E.M., Nievergelt J., Deo N., "Combinatorial Algorithms. Theory and Practice". Prentice-Hall, Inc., 1977.

2. Krichevsky R., "Universal Compression and Retrieval", Kluwer Academic Publishers, 1994.

3. Cover T.M. "Enumerative Source Encoding". IEEE Trans. Inform Theory, vol. IT-19, pp. 73-77, Juan. 1973.

4. Lynch T. Y. Sequence time coding for data compression.// Proc. IEEE, v.54, pp.1490-1491, 1966.

5. Davisson L.D. Comments on "Sequence time coding for data compression".// Proc. IEEE, v.54, p.2010, 1966.

6. Babkin V.F. "A method of universal coding with non-exponent labour consumption" Probl. Inform. Transmission, v.7, pp. 13-21, 1971.

7. Aho A.V., Hopcroft L.E., Ullman J.D. "The Design and Analysis of Computer Algorithms". Addison-Wesley. Publishing Company,1976.

8. Knuth D.E. "The art of computer programming." Vol.2. Addison Wesley, 1981. 